\title{
Treatment Outcomes of Rare Retromolar Trigone Squamous Cell Carcinoma Using Combined Modalities
}

Muhammad Faisal ${ }^{1}$, Taskheer Abbas ${ }^{2}$, Usman Khaleeq ${ }^{2}$, Mohammad Adeel ${ }^{3}$, Abdul Wahid Anwer $^{4}$, Raza Hussain ${ }^{5}$, Arif Jamshed ${ }^{6}$

1. Department of Surgical Oncology, Shaukat Khanum Memorial Cancer Hospital and Research Center, Lahore, PAK 2. Radiation Oncology, Shaukat Khanum Memorial Cancer Hospital and Research Center, Lahore, PAK 3. Department of Surgical Oncology, Shaukat Khanum Memorial Cancer Hospital and Research Center, Lahore, Pakistan 4. Department of Surgical Oncology, Shaukat Khanum Memorial Cancer Hospital and Research Center, Lahore, Pakistan, Lahore, PAK 5. Department of Surgical Oncology, Shaukat Khanum Memorial Cancer Hospital and Research Center, Lahore, Pakistan, lahore, PAK 6. Department of Radiation Oncology, Shaukat Khanum Memorial Cancer Hospital and Research Center, Lahore, PAK

$\square$ Corresponding author: Muhammad Faisal, muhammadfaisal5767@outlook.com Disclosures can be found in Additional Information at the end of the article

\section{Abstract}

\section{Background}

Retromolar trigone squamous cell carcinoma is relatively uncommon and due to its complex anatomy has always remained a challenge in terms of loco-regional control and survival. Surgery, radiotherapy, and chemotherapy as combined modalities have been used but high recurrence rates result in poor outcome.

\section{Methods}

We have retrospectively evaluated records of 62 patients treated in Head and Neck Oncology unit of Shaukat Khanum Memorial Cancer Hospital and Research Centre (SKMCH and RC), Lahore, Pakistan from 2004 to 2014 who were included based on the criteria of histopathological proven squamous cell carcinoma of retromolar trigone (RMT) treated with radical intent. Diagnostic workup for all patients involved clinical examination, imaging modalities usually magnetic resonance imaging (MRI), computerized tomography (CT), Orthopantomogram (OPG), and chest x-ray (CXR) to evaluate regional and distant metastasis, respectively. Kaplan-Meier survival curves were used to depict survival.

Received 03/18/2017

Review began 04/18/2017 Review ended 04/26/2017 Published 05/01/2017

(c) Copyright 2017

Faisal et al. This is an open access article distributed under the terms of the Creative Commons Attribution License CC-BY 3.0., which permits unrestricted use, distribution, and reproduction in any medium, provided the original author and source are credited.

\section{Results}

The study was comprised of 36 male and 26 female patients. Treatment modalities used are surgery only $(n=1)$, radiotherapy alone $(n=13)$, radiotherapy followed by surgery $(n=10)$, chemoradiotherapy $(n=16)$, induction chemotherapy followed by concurrent chemoradiotherapy $(n=19)$, induction chemotherapy followed by surgery, and radiotherapy ( $n$ $=2)$. Surgical interventions include wide local excisions $(n=6)$, marginal mandibulectomy $(n=$ 4 ), and segmental mandibulectomy $(\mathrm{n}=4)$. Surgical margins were clear in $54 \%$, close in $38 \%$, and involved in $8 \%$ of patients. AJCC 7th edition showed cT1 8\%, cT2 22\%, cT3 14\%, and cT4 $56 \%$ while pT1 2\%, pT2 3\%, and pT4 8\%. During follow-up, 18\% patients have come up with local recurrence, $22 \%$ showed persistent disease while $9 \%$ have presented with distant metastasis. The five-year and overall survivals are $38 \%$ and $22 \%$, respectively. 


\section{Conclusion}

Retromolar trigone involvement poses many vital structures at risk of involvement. Late presentation results in involvement of masticator space compromising both mouth opening and surgical outcomes. Surgery and radiotherapy have shown comparable results in disease control. Bone invasion has shown poor outcome in terms of loco-regional control and overall survival.

Categories: Oncology

Keywords: retromolar trigone, squamous cell carcinoma, oral cavity tumors

\section{Introduction}

The retromolar trigone (RMT) in the dry mandible is a triangular area bounded by temporal crest on the medial side, anterior border of ramus on the lateral side, and base posterior to the socket for the third molar [1]. Although RMT tumors are uncommon as compared to other oral sub-sites, squamous cell carcinoma is the most common histopathological presentation. Late stage presentation due to the absence of early symptoms and involvement of adjacent sub-sites such as buccal mucosa, anterior faucial pillar, upper and lower alveolus are the characteristic features of RMT squamous cell carcinoma. Bone invasion has resulted in more morbidity and poor outcomes [2-4]. Pain and trismus are delayed symptoms secondary to involvement of adjacent nerves and muscles of mastication [5]. Little has been published regarding the clear guidelines in terms of management with surgery, radiotherapy, and chemoradiation being used either as single modality or in combination. The objective of our study is to share our experience as the only tertiary care high volume center in a developing country with retromolar trigone squamous cell carcinoma for the clinicopathological outcomes, patterns of failure, and the impact of different treatment modalities on survival.

\section{Materials And Methods}

We have retrospectively evaluated records of 66 patients treated in Head and Neck Oncology unit of Shaukat Khanum Memorial Cancer Hospital and Research Centre (SKMCH and RC), Lahore, Pakistan from January, 2004 to December, 2014 who were included in the study based on the criteria of histopathological and radiological diagnosis of squamous cell carcinoma of RMT. As per protocol, exemption from Institutional Review Board of SKMCH and RC has been granted due to retrospective nature of the study. Diagnostic workup including computerized tomography/magnetic resonance imaging (CT/MRI), Orthopantomogram (OPG), and chest $\mathrm{x}$ ray (CXR) was done. All these patients were discussed in MDT before any intervention. Tumors were staged based on TNM system by AJCC 7th edition. Outcomes were measured in terms of overall survival, treatment-related survival, impact of early versus advanced stage on survival, and modes of failure (local, regional or distant).

Treatment was guided based on the extent of the disease, medical condition, and patient's preference. Patients with mucosal disease with no bony involvement were treated with surgery, surgery followed by radiotherapy based on adverse features such as close or positive margin, perineural invasion, and nodal involvement. Patients with locally advanced disease and severe limitation in mouth opening secondary to trismus related to sub mucous fibrosis or involvement of muscles of mastication, underwent radiotherapy with surgery reserved for persistent disease only and neoadjuvant chemotherapy followed by chemoradiotherapy. Those with obvious bony involvement as indicated in CT scan and adequate mouth opening (more than $30 \mathrm{~mm}$ ) were managed by marginal/segmental mandibulectomy and neck dissection.

\section{Statistical analysis}




\section{Cureus}

The data were analyzed using IBM SPSS Statistics version 20 (IBM Corp., Armonk, NY, USA). Kaplan-Meier survival curves were used to assess survival outcomes. Variables related to patients such as age, gender, tumor grade, treatment modalities used, clinical and pathological stage and pattern of failure were evaluated and collected.

\section{Results}

There were 36 male and 26 female patients. The median age at diagnosis was 53 years (Range 18-79 years). The median follow-up was 15 months and median time to recurrence was seven months. Histologically, 27 (43\%) patients were well differentiated, 29 (47\%) were moderately differentiated, and six (11\%) were poorly differentiated. Geographical distribution shows most of the patients were from the province of Punjab $(n=49)$ followed by KPK $(n=9)$, Sindh $(n=1)$, and Balouchistan $(\mathrm{n}=3)$. Risk factors were smoking (35\%), betel quid (30\%), naswar (16\%), and alcohol 3\% (Table 1).

Treatment modalities used were surgery only $(n=1)$, radiotherapy alone $(n=13)$, radiotherapy followed by surgery $(n=10)$, chemoradiotherapy $(n=11)$, induction chemotherapy followed by radiotherapy $(\mathrm{n}=5)$ induction chemotherapy followed by concurrent chemoradiotherapy $(\mathrm{n}=$ $19)$, induction chemotherapy followed by surgery and radiotherapy $(n=3)$. Surgical interventions included wide local excisions $(n=6)$, marginal mandibulectomy $(n=4)$ and segmental mandibulectomy $(n=4)$. Out of these, one patient had local failure in segmental mandibulectomy group while marginal mandibulectomy group had two local and one regional failures. Ipsilateral neck dissection was performed as part of ablative resections and showed high occult metastatic rate (62.5\%). AJCC 7th edition showed cT1 8\%, cT2 22\%, cT3 14\%, and cT4 56\% while pT1 3\%, pT2 3\%, and pT4 9\%. During follow-up, 20\% patients have come up with local recurrence, $24 \%$ showed persistent disease while $10 \%$ have presented with distant metastasis. The five-year and overall survivals are $38 \%$ and $22 \%$, respectively (Figure 1). The three-year survival for patients treated with surgery followed by radiotherapy, radiotherapy only, and chemoradiotherapy are 76\%, 66\%, and 38\% respectively (Figure 2). Further, classifying the patients into early (stage 1 and 2) and advanced (stage 3 and 4) disease, improved survival (75\%) has been reported in advanced RMT tumors as compared to early ones (32\%) (Table 1).

\section{Demographics and clinicopathological features}

\section{Characteristics}

Age

$<40$ yrs

$>40$ yrs

Sex

Male

Female

Risk factors

Smoking

Yes

No
$\mathbf{N}(\%)$

$11(17.7 \%)$

$51(80.6 \%)$

$36(58.1 \%)$

26 (41.9\%)

$22(35.5 \%)$

42 (64.5\%) 


\section{Cureus}

Betel Nut

Yes

19 (30.6\%)

No

$43(69.4 \%)$

Naswar

Yes

$10(16.1 \%)$

No

$52(83.9 \%)$

Alcohol

Yes

$02(3.2 \%)$

No

$10(80 \%)$

Grade

Well

$27(43.5 \%)$

Moderate

$29(46.8 \%)$

Poor

$06(9.7 \%)$

PNI

Yes

$02(20 \%)$

No

$10(80 \%)$

LVI

Yes

$01(10 \%)$

No

$06(90 \%)$

Recurrence pattern

Local

$12(19.4 \%)$

Regional

$06(9.6 \%)$

Treatment Modality

Surgery

$01(1.6 \%)$

Surgery + Radiotherapy

$10(16.1 \%)$

Radiotherapy

$13(20.9 \%)$

Chemoradiotherapy

$16(25.8 \%)$

Induction chemo + surgery + radiotherapy

$03(4.8 \%)$

Induction chemo + Concurrent chemo radiotherapy

$19(30.6 \%)$

\section{TABLE 1: Demographics and clinicopathological features}




\section{Cureus}

LVI: Lymphovascular invasion; PNI: Perineural invasion.

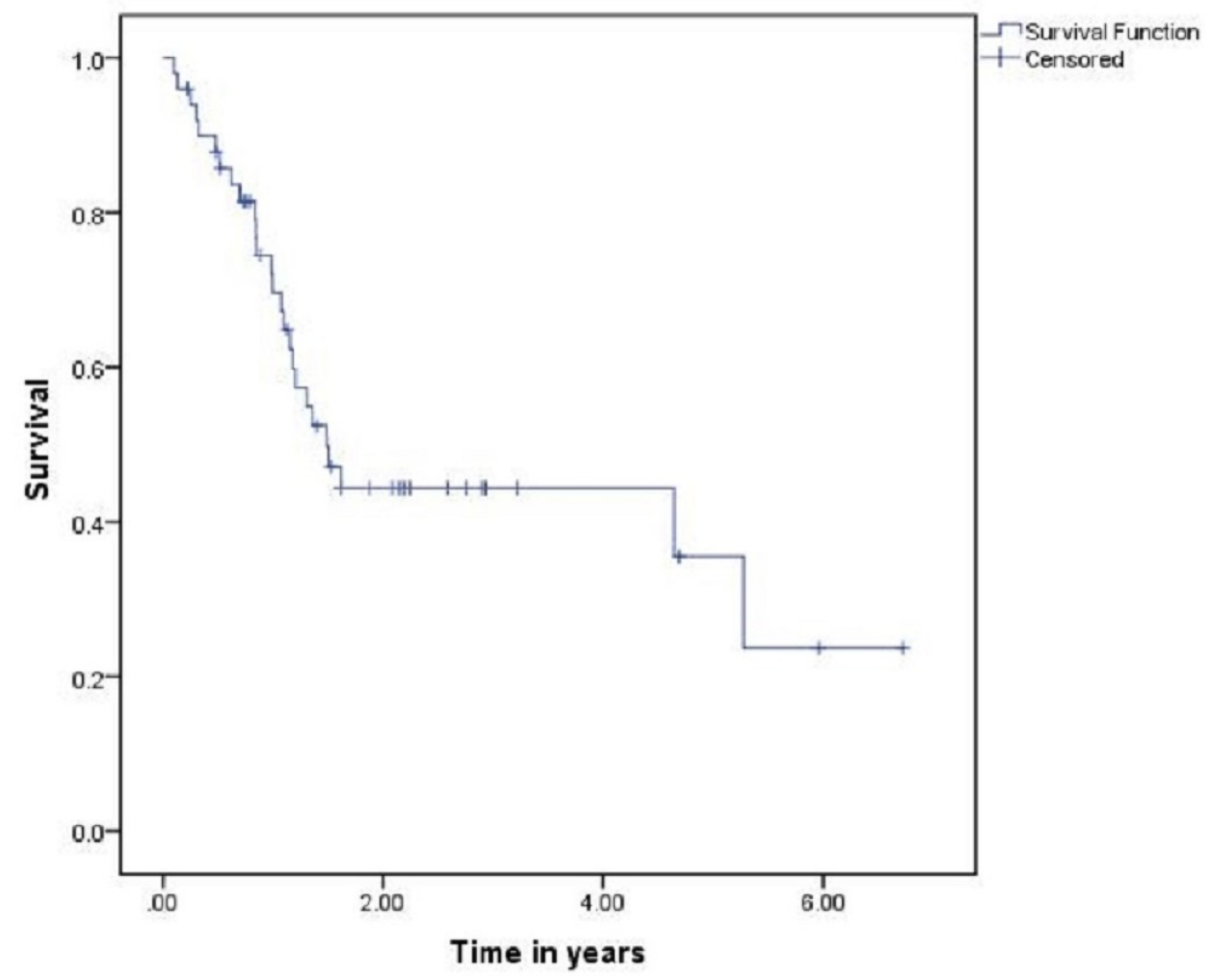

FIGURE 1: Overall survival 


\section{Cureus}

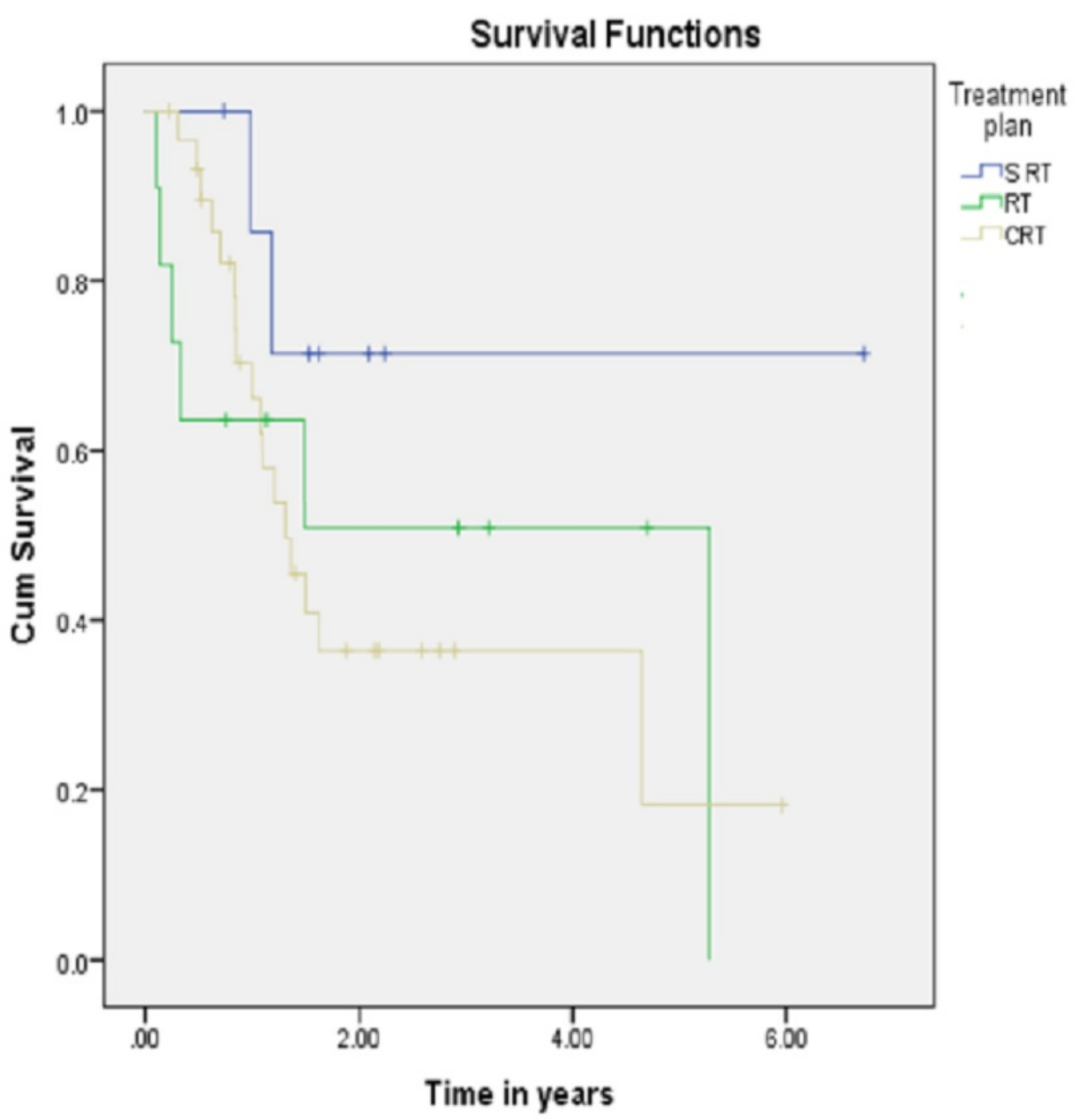

FIGURE 2: Treatment modality related survival

CRT: Chemoradiotherapy; RT: Radiotherapy; S RT: Surgery followed by radiotherapy.

\section{Discussion}

Oral cancers are among the 10 most common causes of cancer-related deaths in the world. The etiology appears to be multifactorial and strongly related to lifestyle, mostly habits, and diet (particularly tobacco alone or in betel, and alcohol use), although other factors as immune defects or immunosuppression, defects of carcinogen metabolism, or defects in DNA-repair enzymes may be implicated. Human papillomavirus (HPV) infection has also been connected with malignant tumors of the oropharyngeal - retromolar trigone junction, together with the other traditional risk factors [6]. PubMed search with the filter "Retromolar trigone, Squamous cell carcinoma" has yielded 112 results which were further filtered out to include 25 articles strictly relating to Retromolar trigone. Retromolar trigone tumors are rare but more aggressive with poor outcome. There is less published data and mostly is retrospective in nature due to rarity of RMT tumors. The peculiar feature of RMT tumors is close proximity to surrounding structures, early involvement of adjacent sub-sites and underlying bone and significant functional compromise post-surgery and radiotherapy [7]. Tumors involving RMT can extend to involve muscles of mastication, soft palate, tonsillar pillars, floor of mouth, and parapharyngeal space. Clinical examination needs to be supplemented by imaging to figure out the sub-clinical spread of the disease. Contrast-enhanced CT has shown high accuracy for 
detection of mandibular invasion [8-9]. Both CT and MRI have their indications in RMT tumors with MRI having better detailing of soft tissue extension, masticator space involvement, perineural spread, and infratemporal fossa invasion.

For early lesion with soft tissue involvement sparing bone, wide local excision seems a reasonable option but this is not a usual happening in these tumors. Bone involvement mandates bony resection either marginal or segmental depending on the extent of the disease.

We have performed wide local resections for early disease with no clinical or radiological bone involvement, marginal and segmental mandibulectomy depending on the extent of bone invasion and ipsilateral neck dissections for all. Literature review has shown comparisons between single versus multimodality treatment protocols.

Barbosa in 1959 has reported the 'retromolar operation' comprising mandibulectomy with enbloc removal of pterygoids and masseter along with ipsilateral neck dissection. A comparison has also been made between surgery and radiotherapy but a small number of patients and evolution of radiation techniques had questioned the final verdict of this study [10]. Kowalski, et al. have analyzed 114 patients with RMT over 31 years with five-year disease-free and overall survival of $48.9 \%$ and $55.3 \%$. He suggested a more extensive approach towards resection and radiotherapy for advanced stage disease and adverse features such as perineural invasion (PNI)/Lymphovascular invasion (LVI).

Pascoal, et al. [11] compared 20 patients undergoing marginal mandibulectomy with 22 treated with segmental mandibulectomy resulting in a failure rate of $35 \%$ and $36.4 \%$, respectively. The survival rate in the group treated with marginal mandibulectomy was $55 \%$ and for group managed with segmental mandibulectomy was $45 \%$ showing no significant difference in terms of local recurrences and survival. We have treated one patient with surgery only showing no loco-regional recurrence, 10 patients with surgery followed by radiotherapy with four patients having local recurrences and one with regional failure. Out of eight patients undergoing mandibulectomy, four patients had marginal mandibulectomy with two local and one regional failure while four had segmental mandibulectomy with only one local failure. Ipsilateral neck dissection has been incorporated as a part of the procedure showing occult metastasis in $62.5 \%$ of these patients involving multiple levels of nodes.

Ayad, et al. have reported the use of radiotherapy for small RMT tumors with curative intent in their series of 46 patients advocating its use in advanced stages without bone invasion [12]. Deo, et al. have emphasized on the use of aggressive surgical approach followed by radiotherapy for better outcome rather than using radiotherapy alone [13]. Comparable outcome has been documented by Hitchcock, et al. using surgery followed by RT versus RT alone but more complications related to the former group [14]. Huang, et al. have reviewed 65 patients with a survival rate of $96 \%, 45 \%$, and $31 \%$ with surgery only, surgery and post-operative radiation, and radiation only respectively during five-year follow-up [15]. In our experience, three-year survival of patients treated with surgery and radiotherapy was $76 \%$ as compared to those treated with RT only which is $66 \%$. Among those managed by radiotherapy only, four patients showed persistent disease and two had regional failures.

Various phase III studies and recent meta-analysis have advocated concurrent chemotherapy and RT for advanced stage III-IV head and neck carcinoma [16-18]. Scher, et al. have demonstrated $37 \%$ five-year loco-regional control and 70\% freedom from distant metastasis in their study on a group of 73 RMT tumor patients [19]. Our results have shown 19 patients treated with induction chemotherapy followed by radiotherapy with a three-year survival of $39 \%$. Out of these, four patients had local failure and six had persistent disease. Concurrent chemo-radiotherapy was offered to five patients with a three-year survival of 38\% (Figure 2). 


\section{Cureus}

The three-year survival of surgically treated patients with clear, close, and involved margin was $100 \%, 63 \%$, and 23\%, respectively. Binahmed, et al. have shared their experience of RMT tumors in 88 patients with the survival of $68 \%, 83 \%$, and $0 \%$ for clear, close, and involved margins [20].

Lymph nodal involvement is a poor prognostic factor in these patients with previous studies reporting nodal involvement to be between 26 and 80\% [21]. Most of the oral cavity tumors have a predictable pattern of drainage to level I-III more commonly involving level IB, RMT tumors usually involve level II and occasionally peri-parotid and retropharyngeal nodes.

Currently, no consensus has been developed as far as the treatment guidelines are concerned. Previous studies have certain limitations such as retrospective nature, limited number of patients due to rarity of the disease. Mendenhall, et al. reported five-year overall survivals of $40 \%$ and $56 \%$ in 35 patients managed with radiation alone and 64 managed with surgery and radiotherapy, respectively [22]. Common practice involves treatment of small tumors (stages I and II) with radiotherapy or surgery and larger tumors (stages III and IV) with combination therapy or surgery alone [23-24]. Early stage disease (stage 1 and 2) has shown improved survival when compared with advanced stage disease (stage 3 and 4) as depicted in Figure 3.

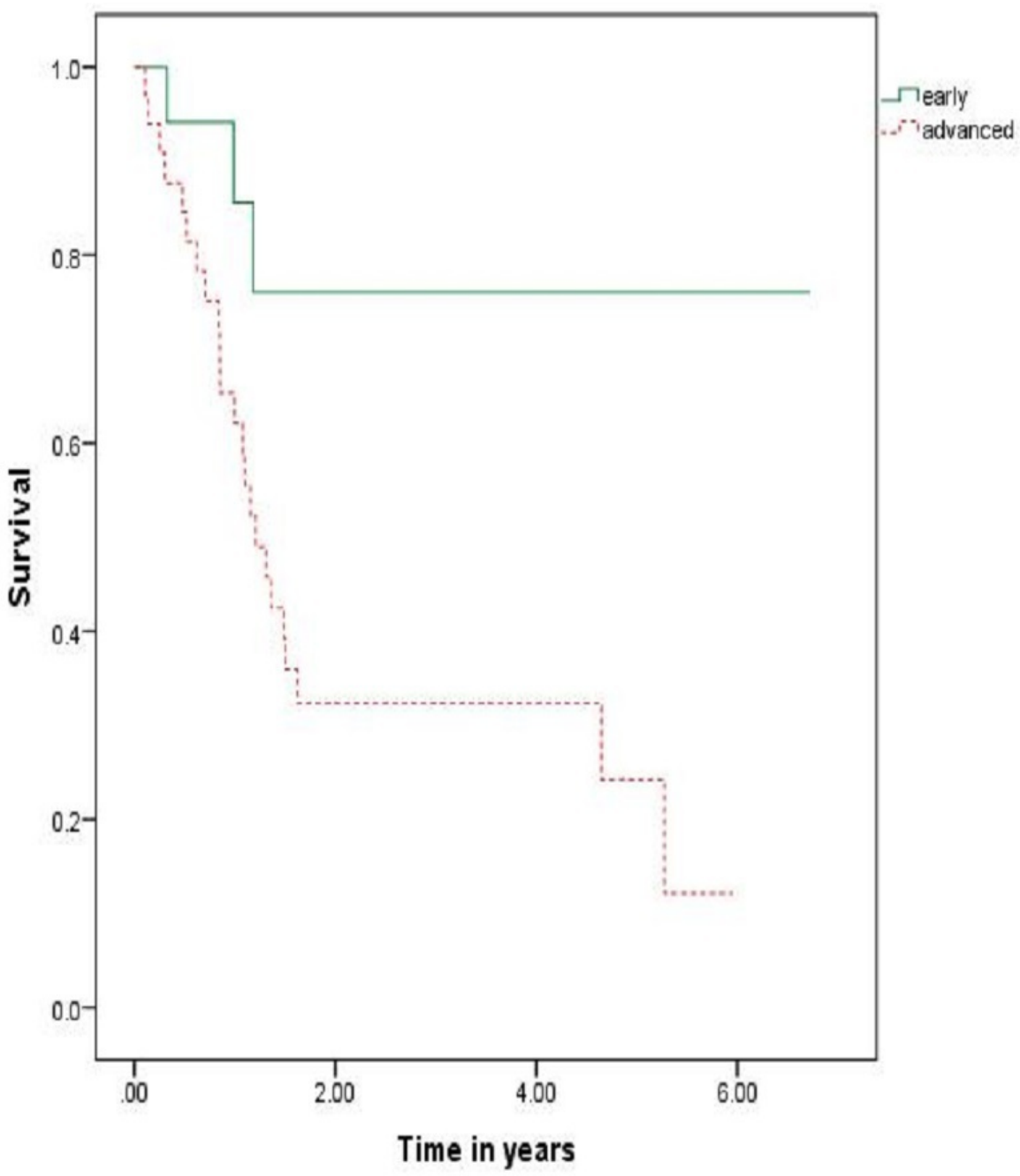

FIGURE 3: Survival related to early and advanced disease 
Our study has certain limitations such as retrospective nature and small sample size. There is a need to gather more uniform population-based data and multicenter analysis before commenting on the guidelines and determining prognostic indicators.

\section{Conclusions}

RMT squamous cell carcinoma is a rare entity with late presentation and usually in advanced stage results in poor outcome. These tumors have high risk of occult metastasis so elective neck dissection is mandatory for regional control. Close and involved surgical margins are related to local failure and adverse survival. Surgery followed by radiotherapy has shown improved overall three-year survival when compared with other patterns of treatment.

\section{Additional Information}

\section{Disclosures}

Human subjects: Consent was obtained by all participants in this study. Informed consent obtained. Animal subjects: All authors have confirmed that this study did not involve animal subjects or tissue. Conflicts of interest: In compliance with the ICMJE uniform disclosure form, all authors declare the following: Payment/services info: All authors have declared that no financial support was received from any organization for the submitted work. Financial relationships: All authors have declared that they have no financial relationships at present or within the previous three years with any organizations that might have an interest in the submitted work. Other relationships: All authors have declared that there are no other relationships or activities that could appear to have influenced the submitted work.

\section{Acknowledgements}

Thanks to Dr. Arif Jamshed and Dr. Raza Hussain for all their support.

\section{References}

1. McGregor I, McGregor F: Cancer of the face and mouth: Pathology and management for surgeons. Churchill Livingstone, Edinburgh; 1986. 499-510.

2. Antoniadoes K, Lazardidis N, Vahtsevanos K, et al.: Treatment of squamous cell carcinoma of the anterior faucial pillar-retromolar trigone. Oral Oncol. 2003, 39:680-686. 10.1016/S13688375(03)00077-0

3. Kowalski L, Hashimoto I, Magrin J: End results of 114 extended “commando” operation for the retromolar trigone carcinoma. Am J Surg. 1993, 166:374-379. 10.1016/S00029610(05)80336-8

4. Byers R, Anderson B, Schwars E, et al.: Treatment of squamous carcinoma of the retromolar trigone. Am J Clin Oncol. 1984, 7:647-652.

5. Genden E, Ferlito A, Shaha A, et al.: Management of cancer of the retromolar trigone . Oral Oncol. 2003, 39:633-637. 10.1016/S1368-8375(03)00103-9

6. Cobzeanu BM, Popescu E, Costan VV, et al.: Retromolar trigone-oropharynx junction maligns tumor surgery: transmandibular versus oral approach. Rev Med Chir Soc Med Nat Iasi. 2015, 119:119-126.

7. Goldie SJ, Soutar DS, Shaw-Dunn J: The effect of surgical resection in the region of the retromolar trigone. J Plast Reconstr Aesthet Surg. 2006, 59:1263-1268. 10.1016/j.bjps.2006.05.024

8. Mazziotti S, Pandolfo I, D’Angelo T, et al.: Diagnostic approach to retromolar trigone cancer by multiplanar computed tomography reconstructions. Can Assoc Radiol J. 2014, 65:335-344. 10.1016/j.carj.2014.04.001

9. Arya S, Rane P, Sable N, et al.: Retromolar trigone squamous cell cancers: a reappraisal of 16 section MDCT for assessing mandibular invasion. Clin Radiol. 2013, 68:680-688. 10.1016/j.crad.2013.08.001

10. Barbosa JF: Cancer of the retromolar area: a study of twenty-eight cases with the presentation 
of a new surgical technique for their treatment. AMA Arch Otolaryngol. 1959, 69:19-30. 10.1001/archotol.1959.00730030023004

11. Pascoal MB, Chagas JF, Alonso N, et al.: Marginal mandibulectomy in the surgical treatment of tonsil and retromolar trigone tumours. Braz J Otorhinolaryngol. 2007, 73:180-184. 10.1016/S1808-8694(15)31064-8

12. Ayad T, Ge'linas M, Guertin L, et al.: Retromolar trigone carcinoma treated by primary radiation therapy: an alternative to the primary surgical approach. Arch Otolaryngol Head Neck Surg. 2005, 131:576-582. 10.1001/archotol.131.7.576

13. Deo SV, Shukla NK, Kallianpur AA, et al.: Aggressive multimodality management of locally advanced retromolar trigone tumors. Head Neck. 2013, 35:1269-1273. 10.1002/hed.23113

14. Hitchcock KE, Amdur RJ, Morris CG, et al.: Retromolar trigone squamous cell carcinoma treated with radiotherapy alone or combined with surgery: a 10-year update. Am J Otolaryngol. 2015, 36:140-145. 10.1016/j.amjoto.2014.10.005

15. Huang C, Chao K, Tsai J, et al.: Cancer of the retromolar trigone: long-term radiation therapy outcome. Head Neck. 2001, 23:758-763.

16. Adelstein DJ, Lavertu P, Saxton JP, et al.: Mature results of a phase III randomized trial comparing concurrent chemoradiotherapy with radiation therapy alone in patients with stage III and IV squamous cell carcinoma of the head and neck. Cancer. 2000, 88:876-883. 10.1002/(SICI)1097-0142(20000215)88:4<876::AID-CNCR19>3.0.CO;2-Y

17. Pignon JP, Bourhis J, Domenge C, et al.: Chemotherapy added to locoregional treatment for head and neck squamous-cell carcinoma: 3 meta-analyses of updated individual data. Lancet. 2000, 355:949-955. 10.1016/S0140-6736(00)90011-4

18. Browman GP, Hodson DI, Mackenzie RJ, et al.: Choosing a concomitant chemotherapy and radiotherapy regimen for squamous cell head and neck cancer: a systematic review of the published literature with subgroup analysis. Head Neck. 2001, 23:579-589. 10.1002/hed.1081

19. Scher ED, Romesser PB, Chen C, et al.: Definitive chemoradiation for primary oral cavity carcinoma: a single institution experience. Oral Oncol. 2015, 51:709-715. 10.1016/j.oraloncology.2015.04.007

20. Binahmed A, Nason RW, Abdoh AA, et al.: Population-based study of treatment outcomes in squamous cell carcinoma of the retromolar trigone. Oral Surg Oral Med Oral Pathol Oral Radiol Endod. 2007, 104:662-665. 10.1016/j.tripleo.2007.04.004

21. Hao SP, Tsang NM, Chang KP, et al.: Treatment of squamous cell carcinoma of the retromolar trigone. Laryngoscope. 2006, 116:916-920. 10.1097/01.mlg.0000214900.07495.39

22. Mendenhall WM, Morris CG, Amdur RJ, et al.: Retromolar trigone squamous cell carcinoma treated with radiotherapy alone or combined with surgery. Cancer. 2005, 103:2320-2325. 10.1002/cncr.21038

23. Skolnik EM, Campbell JM, Meyers RM: Carcinoma of the buccal mucosa and retromolar area . Otolaryngol Clin North Am. 1972, 5:327-331.

24. Glenn MG, Komisar A, Laramore GE: Cost-benefit management decisions for carcinoma of the retromolar trigone. Head Neck. 1995, 17:419-424. 10.1002/hed.2880170511 